\title{
Chronic Activation of Liver X Receptor Sensitizes Mice to High Cholesterol Diet-Induced Gut Toxicity ${ }^{\text {[ }}$
}

\author{
Wojciech G. Garbacz, Hirdesh Uppal, Jiong Yan, Meishu Xu, Songrong Ren, \\ Donna B. Stolz, Min Huang, and Wen Xie
}

Center for Pharmacogenetics and Department of Pharmaceutical Sciences (W.G.G., H.U., J.Y., M.X., S.R., W.X.), Departments of Cell Biology and Physiology (D.B.S.) and Pharmacology and Chemical Biology (W.X.), University of Pittsburgh, Pittsburgh, Pennsylvania; and Institute of Clinical Pharmacology and Guangdong Provincial Key Laboratory of New Drug Design and Evaluation, Sun Yat-Sen University, Guangzhou, China (M.H.)

Received April 5, 2018; accepted July 16, 2018

\section{ABSTRACT}

Cholesterol is essential for numerous biologic functions and processes, but an excess of intracellular cholesterol can be toxic. Intestinal cholesterol absorption is a major determinant of plasma cholesterol level. The liver $X$ receptor $(L X R)$ is a nuclear receptor known for its activity in cholesterol efflux and reverse cholesterol transport. In this study, we uncovered a surprising function of LXR in intestinal cholesterol absorption and toxicity. Genetic or pharmacologic activation of $\mathrm{LXR} \alpha$-sensitized mice to a high-cholesterol diet (HCD) induced intestinal toxicity and tissue damage, including the disruption of enterocyte tight junctions, whereas the same HCD caused little toxicity in the absence of LXR activation. The gut toxicity in HCD-fed LXR-KI mice may have been accounted for by the increased intestinal cholesterol absorption and elevation of enterocyte and systemic levels of free cholesterol. The increased intestinal cholesterol absorption preceded the gut toxicity, suggesting that the increased absorption was not secondary to tissue damage. The heightened sensitivity to HCD in the HCD-fed $\mathrm{LXR} \alpha$-activated mice appeared to be intestine-specific because the liver was not affected despite activation of the same receptor in this tissue. Moreover, heightened sensitivity to HCD cannot be reversed by ezetimibe, a Niemann-Pick C1-like 1 inhibitor that inhibits intestinal cholesterol absorption, suggesting that the increased cholesterol absorption in LXR-activated intestine is mediated by a mechanism that has yet to be defined.

\section{Introduction}

Cholesterol is essential for many biologic functions, but an excess of cholesterol can be toxic or pathogenic. As such, the homeostasis of cholesterol must be tightly regulated. Cholesterol homeostasis is a complex system of synthesis, metabolism, absorption, and excretion of cholesterol molecules (Maxfield and Tabas, 2005).

The liver X receptors (LXRs) are transcription factors belonging to the nuclear hormone receptor superfamily (Willy et al., 1995). There are two LXR isoforms: LXR $\alpha$ (NR1H3) is enriched in the liver, adipose tissue, intestine, kidney, and macrophages, whereas expression of $\operatorname{LXR} \beta$ (NR1H2) is ubiquitous (Chawla et al., 2001). Oxysterols (oxidized cholesterol), such as 0(S), 22(R), 24(S), 25-, and 27-hydroxy cholesterol and

The work was supported in part by the National Institutes of Health [Grants DK083952, ES023438] and the Chinese Ministry of Education 111 Project Grant [Grant B16047]. W.X. was supported in part by the Joseph Koslow endowed chair professorship from the University of Pittsburgh School of Pharmacy.

https://doi.org/10.1124/mol.118.112672. org.

S This article has supplemental material available at molpharm.aspetjournals.

ABBREVIATIONS: FITC, fluorescein isothiocyanate conjugated; GW3965, 3-[3-[[[2-chloro-3-(trifluoromethyl) phenyl]methyl](2,2-diphenylethyl) amino]propoxy]-benzeneacetic O acid, monohydrochloride; HCD, high-cholesterol diet; LXR, liver X receptor; LXR-KI, VP-LXR $\alpha$ knick-in; NPC1L1, Niemann-Pick C1-like 1; ORO, Oil-red O; PCR, polymerase chain reaction; RCT, reverse cholesterol transport; SEM, scanning electron microscopy; siRNA, small interfering RNA; T0901317, N-(2,2,2-trifluoroethyl)-N-[4-[2,2,2-trifluoro- 1-hydroxy-1-(trifluoromethyl)ethyl]phenyl]- benzenesulfonamide; TJ, tight junctions; TUNEL, terminal transferase dUTP nick-end labeling; WT, wild-type.
24(S), 25-epoxycholesterols, are the endogenous LXR ligands (Mutemberezi et al., 2016). Several synthetic LXR agonists, including T0901317 ( $\mathrm{N}$-(2,2,2-trifluoroethyl)-N-[4-[2,2,2-trifluoro1-hydroxy-1-(trifluoromethyl)ethyl]phenyl]- benzenesulfonamide) and GW3965 (3-[3-[[[2-chloro-3-(trifluoromethyl) phenyl]methyl] (2,2-diphenylethyl) amino]propoxy]-benzeneacetic O acid, monohydrochloride), have also been developed (Komati et al., 2017). LXR $\alpha$ is involved in the control of cholesterol metabolism in the liver and intestine through its regulation of genes involved in cholesterol catabolism, efflux, and absorption. $\mathrm{LXR} \alpha$ has been defined as a master regulator of reverse cholesterol transport (RCT) (Jakobsson et al., 2012). RCT is a coordinated process by which cholesterol is transported from peripheral tissues, including the macrophages, back to the liver, where cholesterol is disposed of by biliary secretion and fecal elimination (Zelcer et al., 2009; Zhao and Dahlman-Wright, 2010). Consistent with the role of LXR in RCT, treatment with the LXR agonist GW3965 led to an antiatherogenic effect in $\mathrm{Ldlr}^{-/-}$and apoE $\mathrm{E}^{-1-}$ mice by reducing the occurrence of atherogenic lesions (Joseph et al., 2002). 
Another important pathway of cholesterol homeostasis controlled by LXR is absorption and sterol excretion via the intestines (van der Veen et al., 2009). Intestinal cholesterol absorption is considered a major determinant of the plasma cholesterol level (Bonamassa and Moschetta, 2013), especially with recent evidence that activation of LXR in macrophages may have a limited contribution to LXR agonist-stimulated RCT (Breevoort et al., 2014). In the intestine, LXR is believed to inhibit cholesterol absorption by its trans-suppression of the major intestinal cholesterol absorption transporter NiemannPick C1-like 1 (NPC1L1), which is the therapeutic target of the cholesterol-lowering drug ezetimibe (Van Heek et al., 1997). Npc1l1 ablation in mice resulted in markedly reduced intestinal cholesterol absorption (Altmann et al., 2004; Davis et al., 2004); however, the notion that LXR inhibits cholesterol absorption by suppressing Npc1l1 is not without controversy. Some studies have suggested that inhibition of Npc1l1 by LXR ligands did not affect cholesterol absorption (Kannisto et al., 2014). Others claimed that LXR $\beta$ activity in the small intestine can promote Npc1l1-independent cholesterol absorption (Hu et al., 2012). Transintestinal cholesterol excretion is facilitated by ATP-binding cassette subfamily G members 5 and 8 (Abcg5 and Abcg8). Both Abcg5 and Abcg8 genes are transcriptionally and positively regulated by LXR (Repa et al., 2002), and induction of RCT by LXR agonists was abrogated in Abg5/8 null mice (Calpe-Berdiel et al., 2008).

In this study, we uncovered a surprising function of $\mathrm{LXR} \alpha$ in intestinal cholesterol absorption and gut toxicity. Genetic or pharmacologic activation of $\operatorname{LXR} \alpha$ increased intestinal absorption of cholesterol. The accumulation of free cholesterol in the small intestine triggers endoplasmic reticulum stress, apoptosis, and necrosis, leading to gut toxicity.

\section{Materials and Methods}

Mice. The creation and characterization of VP-LXR $\alpha$ knock-in mice (LXR-KI) have been previously described (Gong et al., 2009). In brief, the LXR $\alpha$ knock-in targeting construct was generated by placing VP-LXR $\alpha$ cDNA (Uppal et al., 2007) in-frame after the endogenous ATG start codon in the endogenous mouse LXR $\alpha$ locus. VP-LXR $\alpha$ cDNA was constructed from the VP16 activation domain of the herpes simplex virus in-frame fused to the amino terminus of mouse LXR $\alpha$ cDNA. The knock-in was achieved by homologous recombination. Mice used in this study were maintained in the C57BL/6 background. Wildtype (WT) mice of the same genetic background were used as controls.

Treatment with Drugs and High-Cholesterol Diet. When necessary, WT and LXR-KI mice were fed normal chow [Purina ISO Pro Rodent 3000 (General) 5P76C3N; St. Louis, MO] or a highcholesterol diet (HCD) that contains $1.25 \%$ cholesterol and $0.5 \%$ sodium cholate (cat. no. TD.03316; Harlan, Madison, WI) starting at 6 to 7 weeks of age and fed ad libitum for 4-12 days. The presence of cholate in HCD is necessary because increased cholesterol in animal chow without cholate is poorly absorbed (Chiang et al., 1998; Woollett et al., 2004). The chow diet and HCD were almost identical in protein, carbohydrate, and protein composition by percentages of weight. For drug treatment, daily gavages of T0901317 (20 mg/kg) (cat. no. 71810; Cayman Chemical, Ann Arbor, MI), GW3965 (20 mg/kg) (cat. no. 10054, Cayman), or ezetimibe (10 mg/kg) (cat. no. SML1629 from Sigma-Aldrich, St. Louis, MO) were given. Ezetimibe treatment started 2 days before the introduction of HCD.

Study Approval. The Central Animal Facility of the University of Pittsburgh is fully accredited by American Association for Laboratory Animal Care. All procedures were performed in accordance with relevant federal guidelines and with the approval of the University of Pittsburgh Institutional Animal Care and Use Committee.

Body Composition Analysis and Serum and Liver Biochemistry. Fat mass, lean mass, and water mass were measured by EchoMRI analyzer (Houston, TX). Serum levels of triglycerides, total cholesterol, alanine transaminase, aspartate transaminase (Stanbio Laboratory, Boerne, TX), free cholesterols, and cholesteryl esters (Abcam, Cambridge, MA) were measured by using commercial assay kits. Hepatic lipids were extracted using chloroform/methanol Folch method (Folch et al., 1957) before measuring the cholesterol contents.

Histology, Terminal Transferase dUTP Nick-End Labeling Assay, Filipin, and Oil-Red O Staining and Cholesterol Uptake Assay in Cell Culture. The general histology of the liver and duodenum/proximal jejunum was evaluated by H\&E staining. For the Oil-red O (ORO) staining, liver tissues were dehydrated in 30\% sucrose solution, embedded in optimal cutting temperature compound, sectioned using cryostat, and stained with ORO $(0.5 \%$ in isopropanol) from Sigma-Aldrich. For filipin staining, cryosections were incubated with filipin solution (cat. no. FG765 from Sigma) $(50 \mu \mathrm{g} / \mathrm{ml})$ for 30 minutes, washed with phosphate-buffered saline, glass-covered, and analyzed. Terminal transferase dUTP nick-end labeling (TUNEL) staining was conducted using an assay kit from Roche Diagnostics, Basel, Switzerland) according to the manufacturer's instructions. Cholesterol uptake assay in HCT-116 cells was conducted using an assay kit (cat. no. 600440) from Cayman. Chemical.

Transmission Electron Microscopy and Scanning Electron Microscopy. Transmission electron microscopy (TEM) and scanning electron microscopy (SEM) of the duodenal tissues was performed in blind as previously described (Nakao et al., 2003). Chosen images show representative areas.

In Vivo Cholesterol Absorption Assay and Fluorescein Isothiocyanate Conjugated-Dextran Mediated Intestinal Permeability Assay. For in vivo cholesterol absorption assay, mice were gavaged with $5 \mu \mathrm{Ci}$ of $\left[1,2-{ }^{3} \mathrm{H}(\mathrm{N})\right]$-cholesterol (PerkinElmer, Waltham, MA) together with $0.1 \mathrm{mg}$ of unlabeled cholesterol in $100 \mu \mathrm{l}$ corn oil. Mice were killed 2 hours later, and plasma was collected and analyzed by liquid scintillation counting. The percentage of cholesterol absorption was calculated as follows: percent cholesterol absorption $=\left[{ }^{3} \mathrm{H}\right]$ in plasma $/\left[{ }^{3} \mathrm{H}\right]$ dosing $\times 100$. Total radioactivity in the plasma was calculated based on the assumption that mice possess approximately $4.0 \mathrm{ml}$ of plasma per $100 \mathrm{~g}$ of body weight (Mitrura and Rawnsley, 1981). For intestinal permeability assay, mice were fasted overnight and gavaged with fluorescein isothiocyanate conjugated dextran (FITC-dextran) (average molecular weight, 3000-5000 Da, cat. no. 46944 from Sigma Aldrich) diluted in phosphate-buffered saline $(100 \mathrm{mg} / \mathrm{ml})$ for the final dose of $44 \mathrm{mg} / 100 \mathrm{~g}$ of body weight. Four hours later, blood was sampled from the mice tails. The serum samples were collected and analyzed by fluorescent plate reader (excitation, 485; absorption, $528 \mathrm{~nm}$ ) (Calon et al., 2007).

LXR RNA Interference. The human LXR small interfering RNAs (siRNAs) and the scrambled controls were purchased from Qiagen (Valencia, CA). The sequences are as follow: $\operatorname{siLXR} \alpha$ (59-AGCAGGGCUGCAAGUGGAA-39) and siLXR $\beta$ (59-CAGAUCCG G-AAGAAGAAGA-39). The siRNA transfection was conducted using LipofectAMINE 2000 (Invitrogen, Carlsbad, CA) as we have previously described (Ou et al., 2014). The siRNAs were added to the final concentration of $20 \mathrm{nM}$ during transfection. HCT116 colon cancer cells were cultured overnight, followed by treatment with vehicle or $1 \mu \mathrm{M}$ GW3965 overnight, and then followed by 24 hours of incubation with NBD-cholesterol.

Real-Time Polymerase Chain Reaction and Western Blot Analysis. Total RNA was extracted using the Trizol reagent from Invitrogen. Real-time polymerase chain reaction $(\mathrm{PCR})$ was performed using SYBR Green reagent using the ABI 7300 real-time PCR system. Cyclophilin was used as a housekeeping gene internal control. For Western blot analysis, $30 \mu \mathrm{g}$ of protein extracts from tissue lysate were 
separated on SDS-PAGE gels and transferred onto polyvinylidene fluoride membrane. For duodenum/proximal jejunum, villus and crypt cells were scraped from the intestine during tissue harvest. The primary antibodies used were anti-human $\operatorname{LXR} \alpha$ (cat. no. PP-PPZ0412-00), anti-human LXR $\beta$ cat. no. PP-K8917-00) from Perseus Proteomics (Tokyo, Japan); anti-Cyp7A1 (cat. no. sc-25536; Santa Cruz Biotechnology, Inc., Dallas, TX); anti-phospho-eIF2 $\alpha$ (Ser51) (cat. no. 9721), anti-eIF2 $\alpha$ (cat no. 9722), and ant-CHOP (cat no. 5554) from Cell Signaling Technology (Danvers MA); anti-NPC1L1 (cat. no. PA1-116800) from Thermo-Fisher Scientific (Waltham, MA); and anti- $\beta$-actin (cat. no. A1978) from Sigma.

Statistics. GraphPad Prism software (San Diego, CA) was used for statistical analysis. The unpaired Student $t$ test was performed without correction for multiple comparisons. Statistical significance threshold was set at $P<0.05$. Data represent mean \pm S.D.

\section{Results}

Activation of LXR Sensitizes Mice to HCD-Induced Toxicity. Acute activation of LXR is known to promote cholesterol efflux and RCT (Zelcer et al., 2009; Zhao and Dahlman-Wright, 2010). We used the VP-LXR $\alpha$ knock-in (LXR-KI) mice to study the effect of chronic activation of LXR $\alpha$ on cholesterol homeostasis, especially when the mice are challenged with an HCD. The VP-LXR $\alpha$ cDNA was constructed by fusing the VP16 activation domain of the herpes simplex virus to the amino terminus of mouse LXR $\alpha$ sequence. The LXR-KI mice were created by knock-in the
VP-LXR $\alpha$ cDNA in-frame and immediately after the endogenous ATG start codon of the mouse LXR $\alpha$ locus. As a result, VP-LXR $\alpha$ will be expressed under the control of the endogenous $\mathrm{LXR} \alpha$ promoter, whereas the WT LXR $\alpha$ allele will be disrupted in the homozygous LXR-KI mice (Gong et al., 2009). The expression of VP-LXR $\alpha$ and ablation of the endogenous LXR $\alpha$ in the liver and intestine were confirmed by Northern blot analysis (Fig. 1A).

Having expected an increased cholesterol disposal in LXR-KI mice, we did not expect to find that the male LXR-KI mice exhibited signs of toxicity when fed an HCD. These included hunched posture (Fig. 1B) and a marked loss of body weight (Fig. 1C) compared with the WT controls. The female LXR-KI mice also showed heightened sensitivity to HCD-induced toxicity (data not shown). The toxic effect was HCD-dependent because LXR-KI mice maintained on a chow diet exhibited no difference in body weight compared with their WT counterparts (data not shown). A similar heightened sensitivity to HCD-induced toxicity was observed in HCD-fed mice treated with the selective LXR agonist GW3965 (Fig. 1C). Body composition analysis by magnetic resonance imaging showed that both the fat mass and lean mass were lower in the HCD-fed LXR-KI mice (data not shown). Food consumption did not differ between the two genotypes (Fig. 1D). Another surprising finding is that after a 12-day HCD feeding, the total cholesterol level in the serum of LXR-KI mice was elevated (Fig. 1E).
A

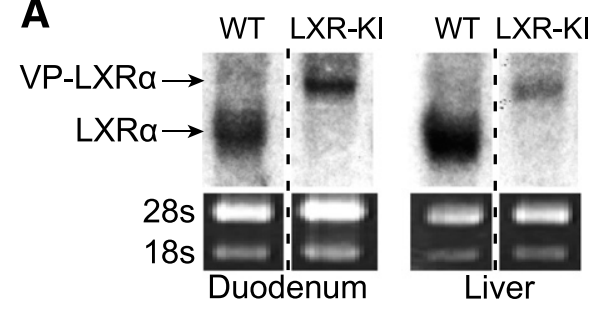

C
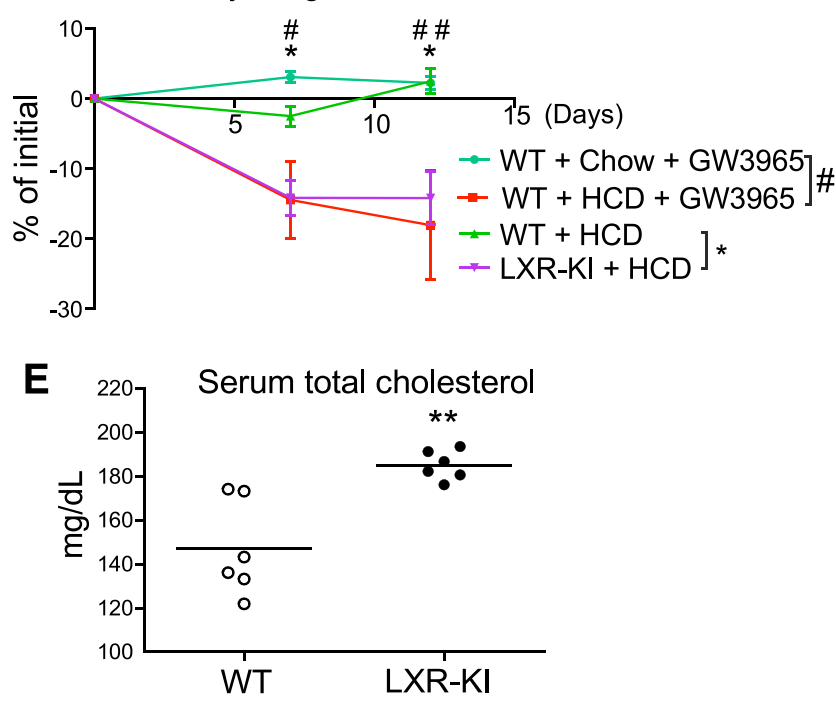

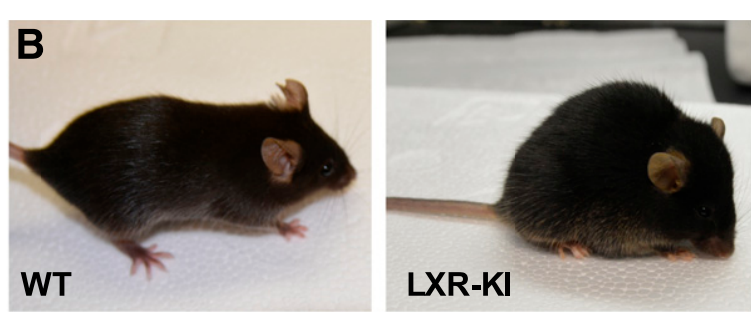

D

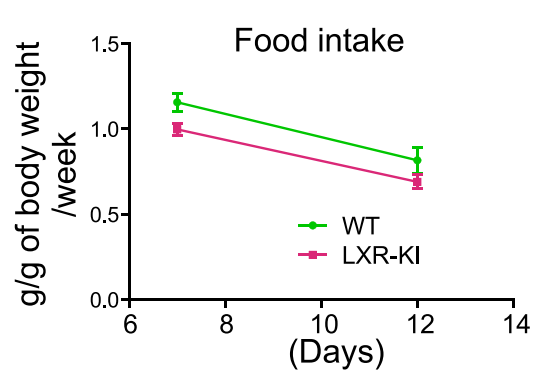

Fig. 1. Activation of LXR sensitizes mice to HCD-induced toxicity. (A) Expression of VP-LXR $\alpha$ and ablation of the endogenous LXR $\alpha$ in the liver and intestine of LXR-KI mice were confirmed by Northern blot analysis. (B) Appearance of WT and LXR-KI male mice fed an HCD for 12 days. (C) Time course of body weight changes in WT and LXR-KI mice fed an HCD: WT fed chow or HCD and treated with GW3965 (20 mg/kg per day) ( $n=6$ to 7) (D) Time course of food intake in WT and LXR-KI mice fed HCD for 12 days. (E) Serum levels of total cholesterol after feeding with HCD for 12 days. ${ }^{*} P<0.05 ;{ }^{*} P<0.01$, LXR-KI vs. WT under HCD; ${ }^{*} P<0.05 ;{ }^{\# \#} P<0.01$, GW3965-treated WT mice, chow vs. HCD. 

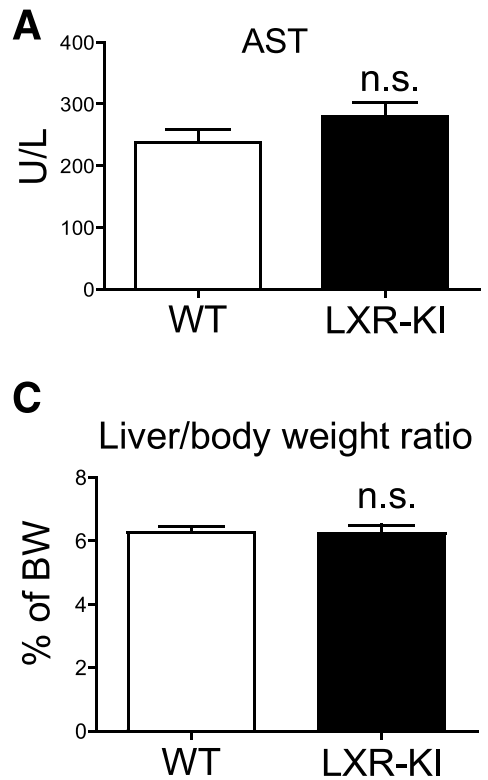

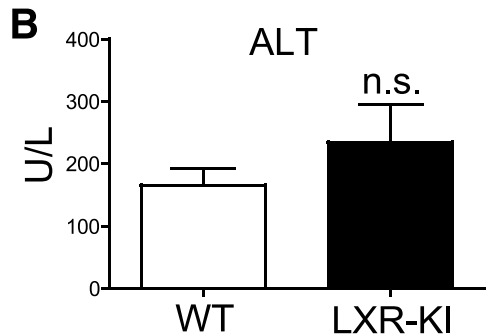

D

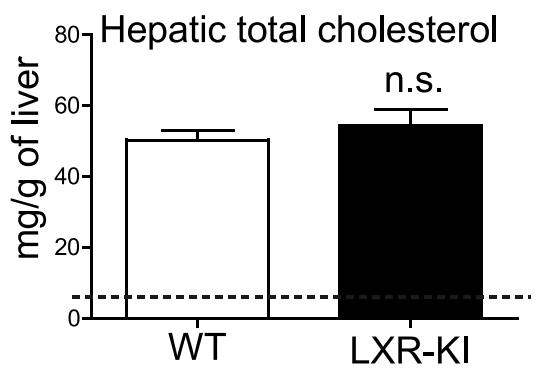

E
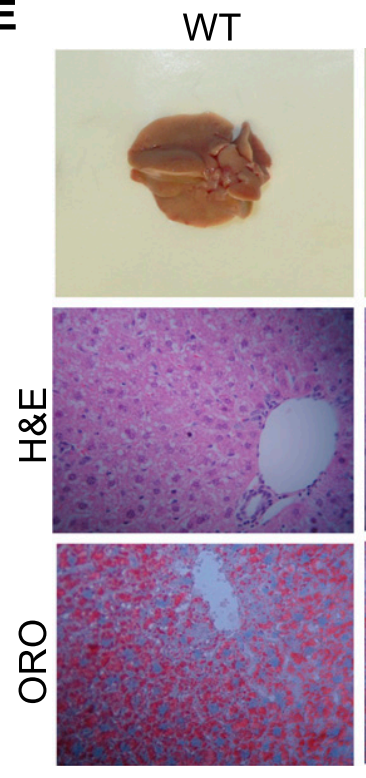
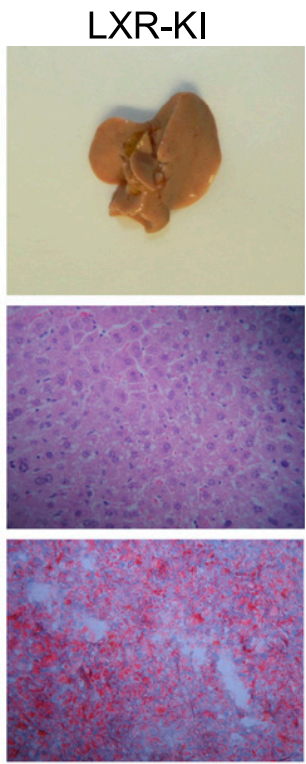

Fig. 2. HCD-induced toxicity in LXR $\alpha$ activated mice is not associated with liver toxicity. WT and LXR-KI male mice were fed an HCD for 12 days. (A and B) Serum levels of aspartate transaminase (AST) (A) and alanine transaminase (ALT) (B). (C) Liver-to-body ratio. (D) Liver total cholesterol levels. The dashed line represents average level of total liver cholesterol in chow fed mice. (E) Macroscopic appearance (top panel) and histology by H\&E (middle panel) and ORO staining (bottom panel) of the livers. $n=6$ to 7 . n.s., statistically not significant.

HCD-Induced Toxicity in LXR $\alpha$ Activated Mice Is Not Associated with Liver Toxicity. Since LXR $\alpha$ is highly expressed in the liver and the liver plays an important role in cholesterol metabolism, we suspected that liver toxicity might be responsible because cholesterol accumulation in the liver may eventually lead to impaired hepatic function (Beltroy et al., 2005). To our surprise, the AST (Fig. 2A) and ALT (Fig. 2B) activities were not different between the HCD-fed WT and HCD-fed LXR-KI mice. The liver-to-body weight ratio were also indistinguishable between the two genotypes (Fig. 2C). HCD feeding for 12 days resulted in approximately a 10-fold increase in hepatic cholesterol levels above the chow-fed levels as expected (Fig. 2D), but cholesterol levels were not different between the two genotypes. Neither the macroscopic appearance nor the histology (H\&E or ORO staining) of the livers of the HCD-fed LXR-KI mice were different from their WT counterparts (Fig. 2E). LXR promotes the biosynthesis of bile acids from cholesterol in mice through its positive regulation of Cyp7a1 (Peet et al., 1998). As expected, hepatic expression of Cyp7a1 increased in the LXR-KI mice (Supplementary Fig. 1A). Upon the HCD feeding, expression of Cyp7a1 in WT mice was suppressed, likely as a result of the activation of FXR by the cholic acid contained in the HCD, but the expression of Cyp7a1 was sustained in the LXR-KI mice because of the constitutive activation of LXR $\alpha$ in these mice (Supplementary Fig. 1A). At the functional level, the serum (Supplementary Fig. 1B) and liver (Supplementary Fig. 1C) levels of total bile acids were higher in the HCD-fed LXR-KI mice, which may have been accounted for by the constitutive upregulation of Cyp7a1 resulting from the constitutive activation of $\operatorname{LXR} \alpha$. Interestingly, the fecal levels of bile acids were lower in the HCD-fed LXR-KI mice (Supplementary Fig. 1D), a phenomenon that remains to be understood. Among the FXR target genes, the ileal expression of fibroblast growth factor 15 (Fgf15), (Supplementary Fig. 1E) and the hepatic expression of small heterodimer partner (Shp) (Supplementary
Fig. 1F) and bile salt export pump (Bsep) (Supplementary Fig. 1G) were mostly upregulated by HCD, likely owing to the cholic acid in the diet, but expression of these genes in HCDfed mice was indistinguishable between the WT and LXR-KI mice genotypes.

Activation of LXR $\alpha$ Leads to Heightened HCDInduced Gut Toxicity, Increased Intestinal Permeability, and Disruption of Intestinal Tight Junctions. LXRs are also known to have their roles in the small intestine, including the downregulation of the cholesterol absorption transporter Niemann-Pick C1-Like 1 (NPC1L1) (Duval et al., 2006) and upregulation of the cholesterol efflux transporters Abcg5 and Abcg8 (Repa et al., 2002). Histologic analysis by H\&E staining showed marked structural disruption and loss of regular villi in the duodenal and proximal jejunum of the LXR-KI mice fed an HCD for 12 days (Fig. 3A, upper panels). SEM revealed detailed lesions or rough surfaces of enterocyte microvilli in the LXR-KI mice, in contrast to the smooth-looking surface of microvilli in the WT mice (Fig. 3A, middle and bottom panels). The intestinal tissue damage in the HCD-fed LXR-KI mice was further confirmed by the TUNEL assay (Fig. 3B). The increased apoptosis in LXR-KI mice was intestine-specific because no TUNEL signals were detected in the liver, kidney, or skeletal muscle of the same mice (Supplementary Fig. 2), despite the expression of VP-LXR $\alpha$ in these tissues (Gong et al., 2009). Increased villus damage and apoptosis were also seen in HCD-fed WT mice treated with the LXR agonist T0901317 (Supplementary Fig. 3)

The gut toxicity in HCD-fed LXR-KI mice was associated with increased intestinal permeability and disruption of enterocyte tight junctions. The intestinal permeability was estimated by measuring the serum level of FITC-dextran after oral gavage of this drug (Calon et al., 2007). As shown in Fig. 3C, although the basal intestinal permeability did not differ between the two genotypes, 12 days of HCD feeding 
A
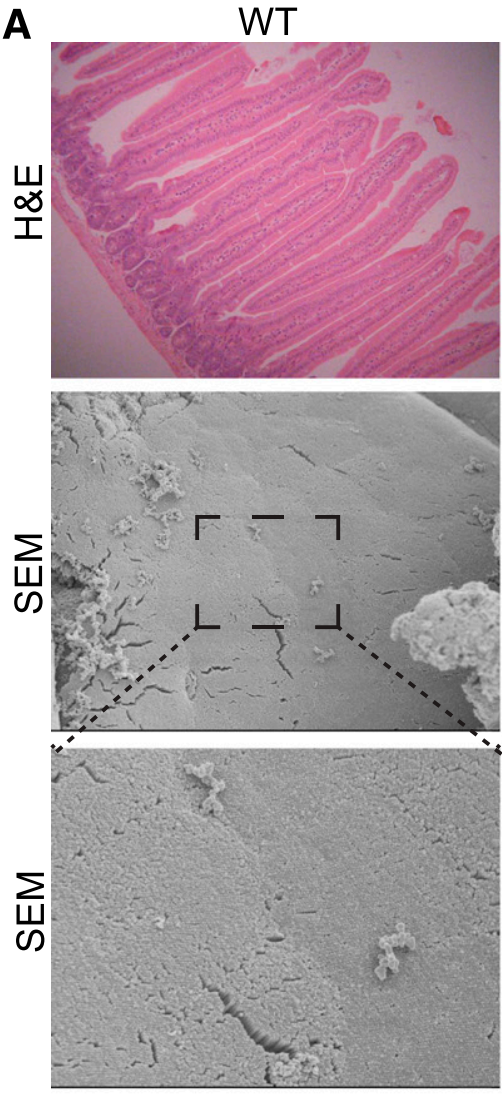

\section{C}

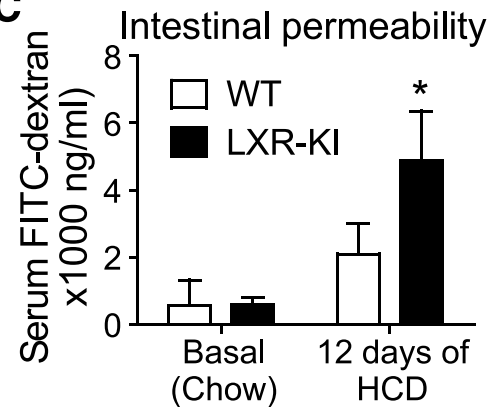

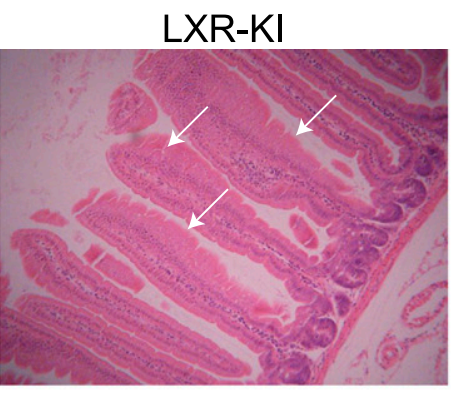
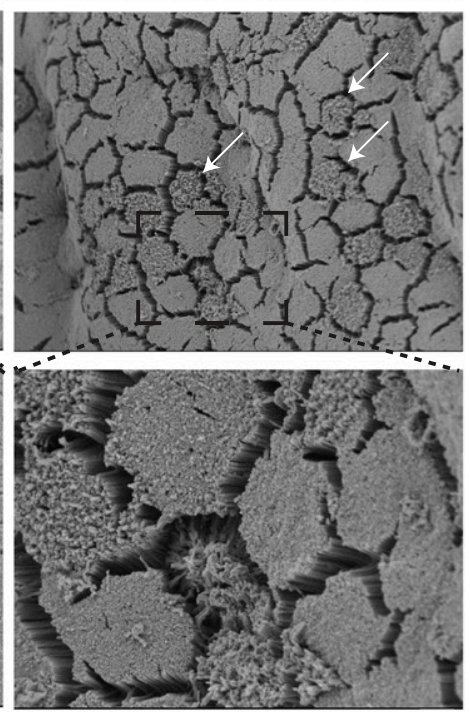

D

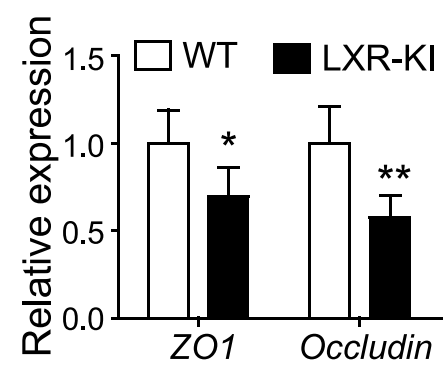

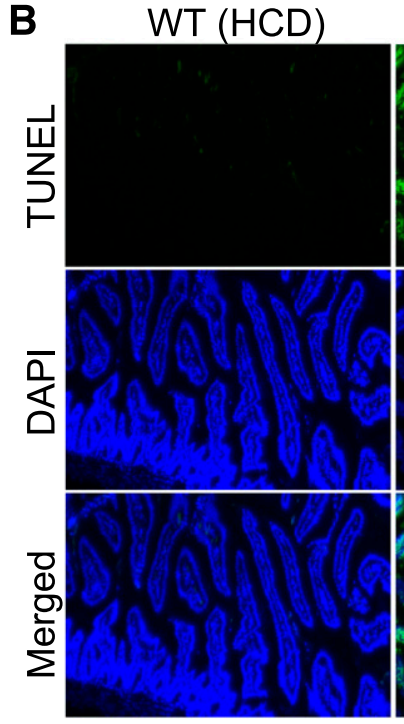
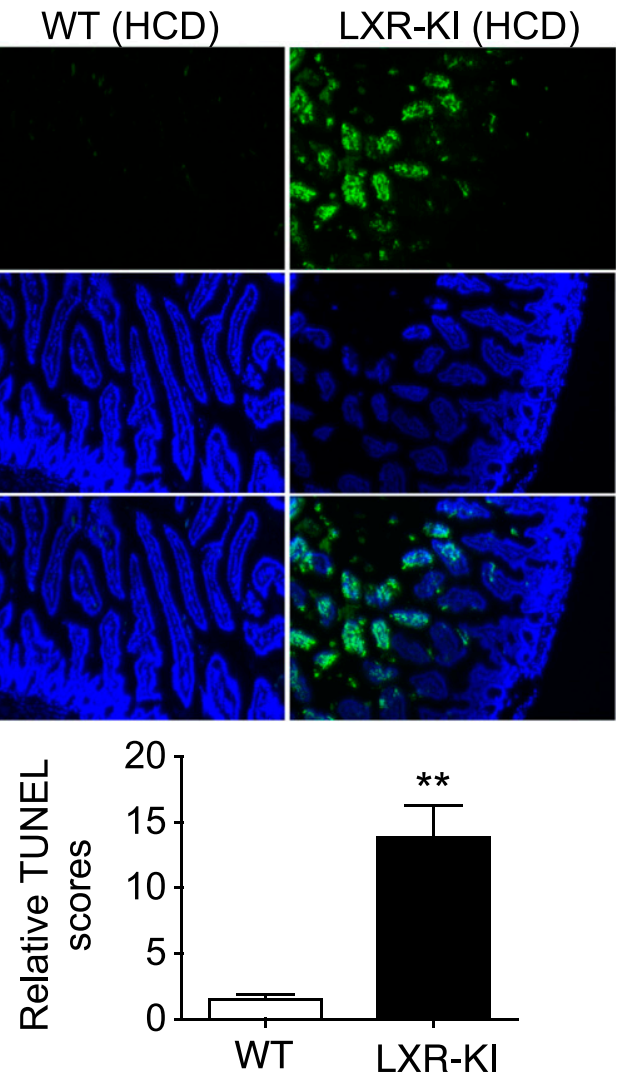

E

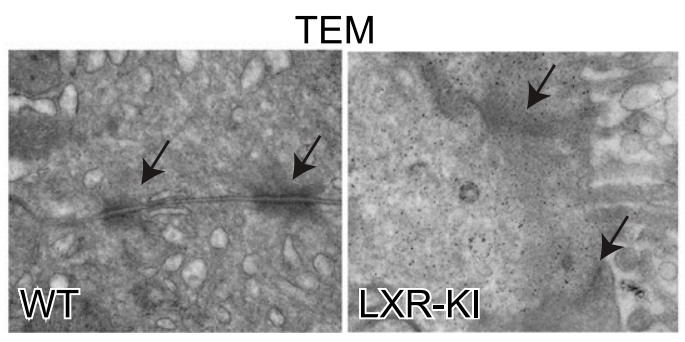

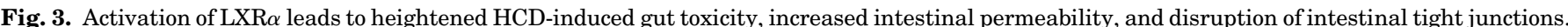

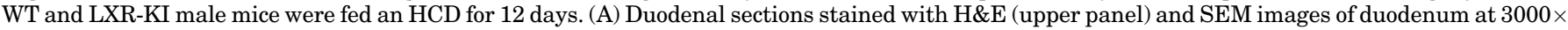

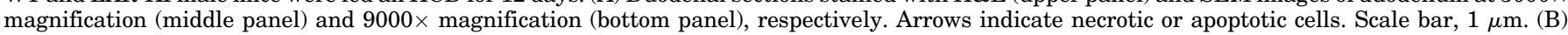

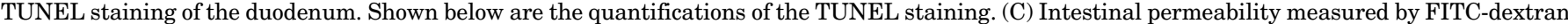

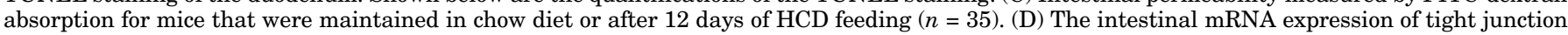

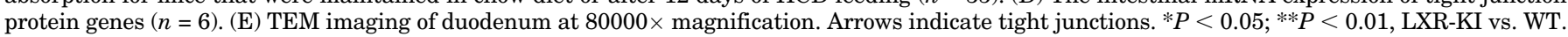

resulted in a higher intestinal permeability in the LXR-KI group. The space between enterocytes of intestinal epithelium is sealed by tight junctions (TJs), which regulate the paracellular flux through the intestinal barrier (Ulluwishewa et al., 2011). At the gene expression level, mRNA expression of Zonula occludens-1 (ZO1) and Occludin, two genes that encode TJ proteins, was decreased in the HCD-fed LXR-KI mice (Fig. 3D). At the histologic level, TEM showed that the structural integrity of the TJ in the HCD-fed LXR-KI mice was compromised compared with their WT counterparts (Fig. 3E).

Activation of LXR $\alpha$ Results in Intestinal and Systemic Accumulation of Free Cholesterol upon HCD Feeding. The higher serum levels of cholesterol in HCD-fed LXR-KI mice prompted us to examine whether activation of
LXR $\alpha$ affects intestinal cholesterol absorption by ORO staining and filipin staining. ORO staining showed a substantial increase in lipids in the intestine of the LXR-KI mice, and most of the ORO signals in LXR-KI mice were localized in the villus region (Fig. 4A). ORO staining detects both cholesterol and triglycerides. LXR $\alpha$ is known to promote intestinal lipogenesis (Peet et al., 1998), but most de novo fatty acid synthesis occurs in the crypt regions rather than the villus cells (Shakir et al., 1978). The predominant villus ORO signals suggested that what accumulated was cholesterol rather than triglycerides, and a direct measurement of duodenal triglyceride levels did not show statistically significant differences between groups (data not shown). We next used filipin staining to confirm the cholesterol identity of the ORO staining. Filipin 
A

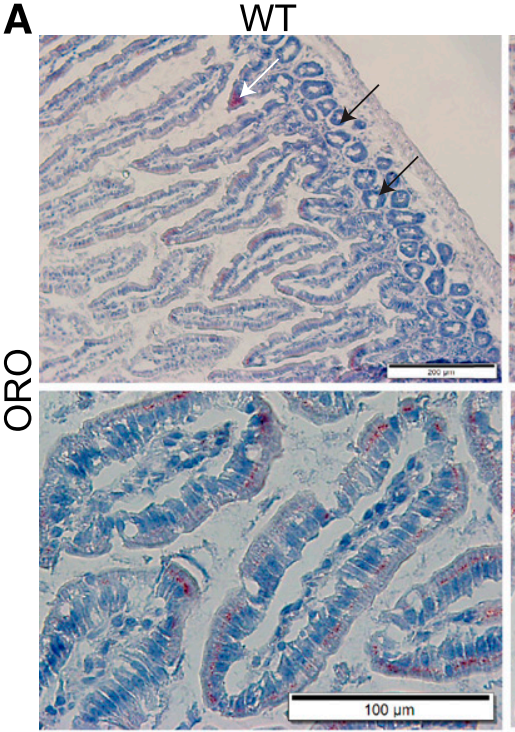

B
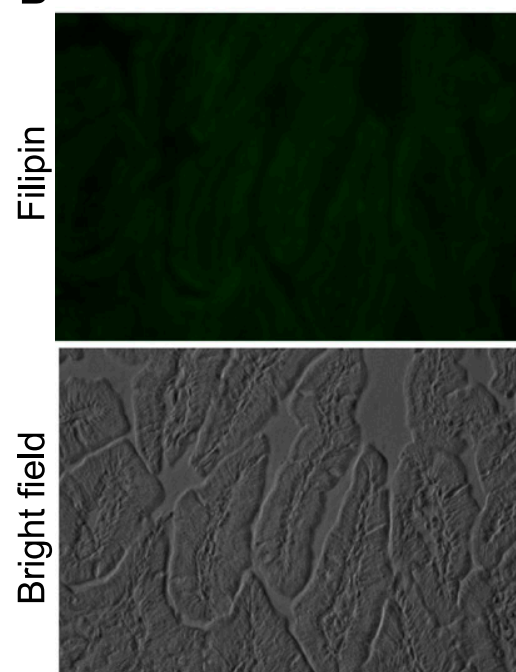

LXR-KI
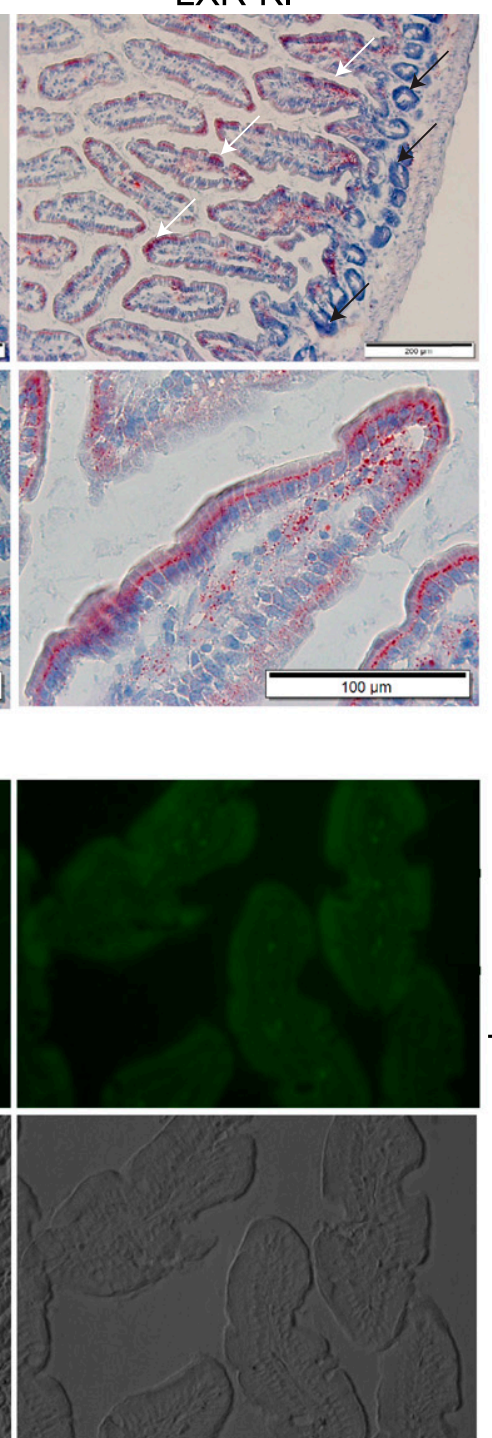

C

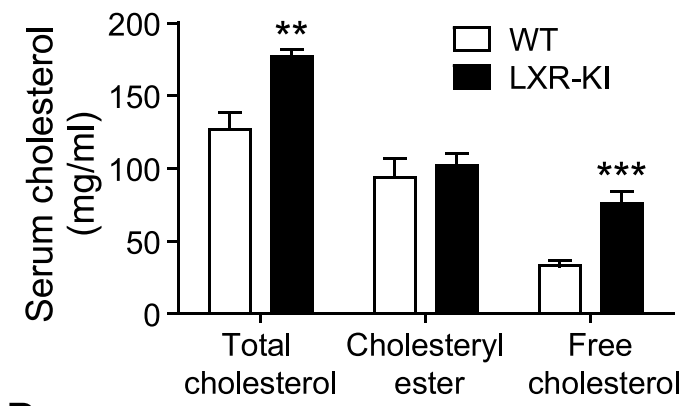

D

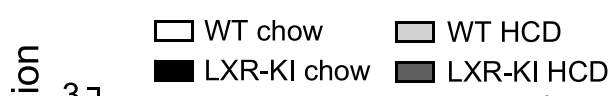

$E$
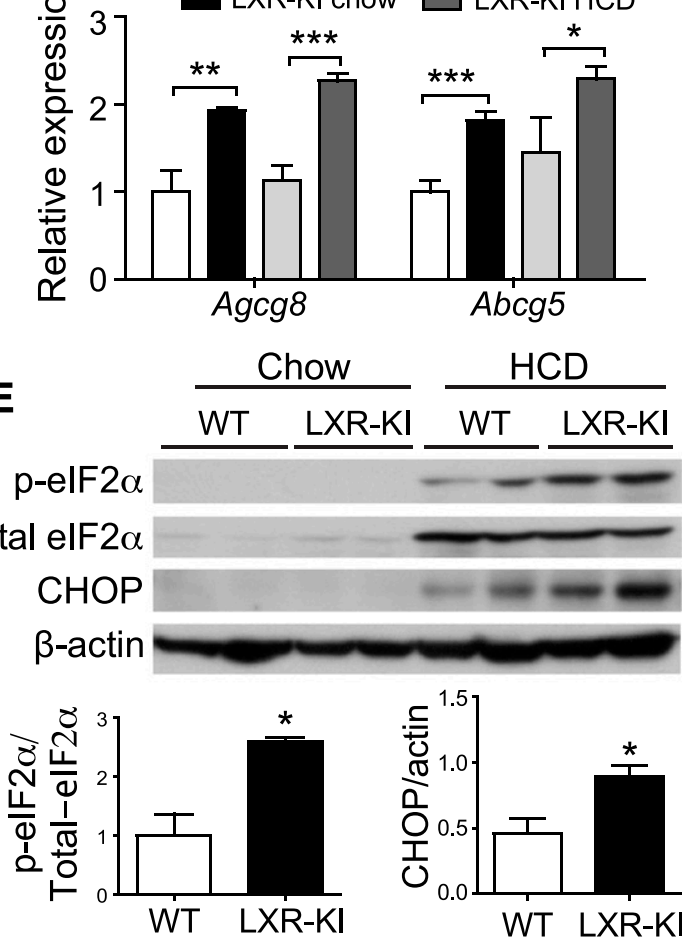

stains specifically for the free form of cholesterol (Kruth, 1984). As shown in Fig. 4B, the filipin staining was more pronounced in the intestine of the HCD-fed LXR-KI mice, suggesting higher levels of intestinal free cholesterol accumulation in this genotype. Higher levels of free cholesterol were also found in the serum of the HCD-fed LXR-KI mice (Fig. 4C). The increased cholesterol uptake in the LXR-KI mice was unexpected because the LXR-KI mice had an elevated expression of the LXR target cholesterol efflux transporters Abcg5 and Abcg8, regardless of the HCD feeding (Fig. 4D). We also measured the intestinal expression of the scavenger receptor class B type 1 (Srb1) and cluster of differentiation $36(\mathrm{Cd} 36)$, both of which are multiligand membrane receptors capable of binding cholesterol (Werder et al., 2001). The HCD feeding decreased and increased the intestinal expression of $\mathrm{Srb1}$ and $C d 36$, respectively, but expression of these two genes did not diff between the WT and LXR-KI, mice regardless of the diet (Supplementary Fig. 4). An excess of free cholesterol is known to cause cellular toxicity by inducing ER stress (Tabas, 2002). Indeed, the protein levels of phosphorylated eIF $2 \alpha$ (Ser51) and CHOP, two ER stress markers, were elevated in the intestine of the HCD-fed LXR-KI mice compared with their WT counterparts (Fig. 4E). The increased free cholesterol and ER stress in the enterocytes may have accounted for the gut and/or systemic toxicity that were observed in the HCD-fed LXR-KI mice.

Activation of LXR $\alpha$ Increases Intestinal Cholesterol Absorption Even in the Presence of the Npc111 Inhibitor Ezetimibe and Precedes the Gut Toxicity. We then directly measured the effect of $\mathrm{LXR} \alpha$ activation on cholesterol absorption by gavaging mice with radio-labeled cholesterol. In the chow-fed mice, the cholesterol absorption 
A
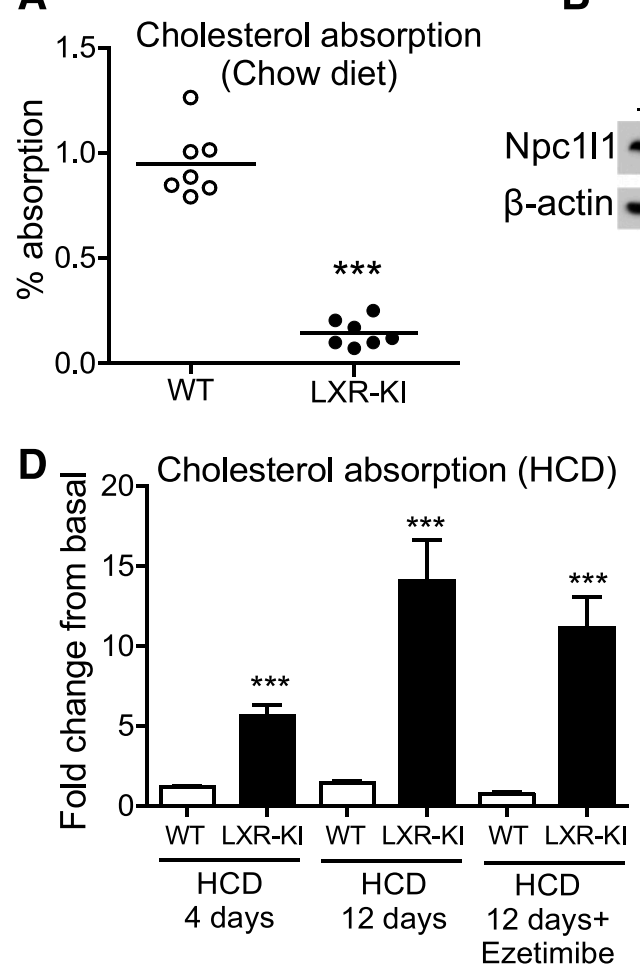

B

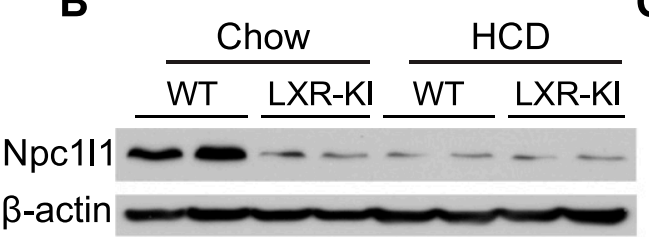

$\mathbf{F}$

C ${ }^{25} \mathrm{j}$ Serum cholesterol (Chow)

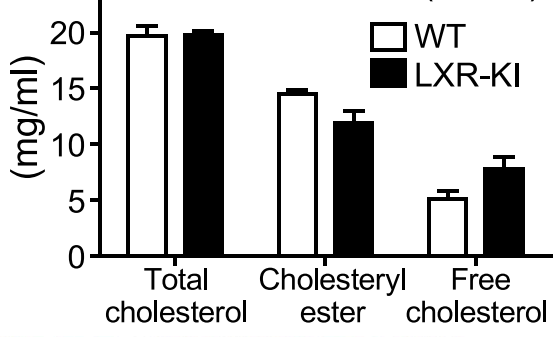

E

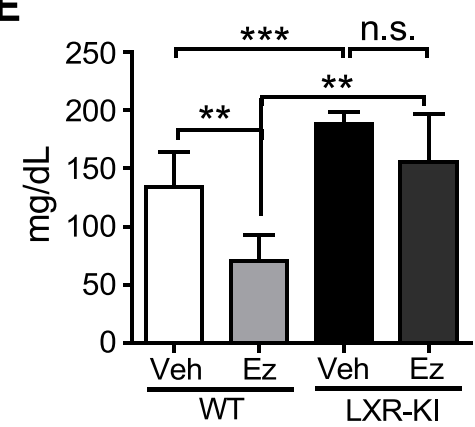

I

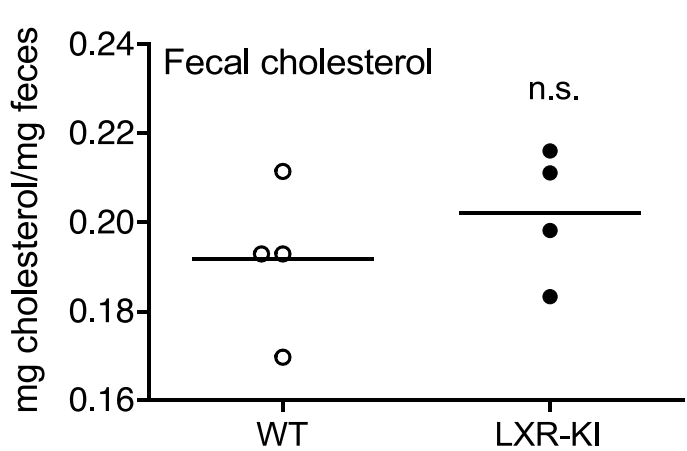

G

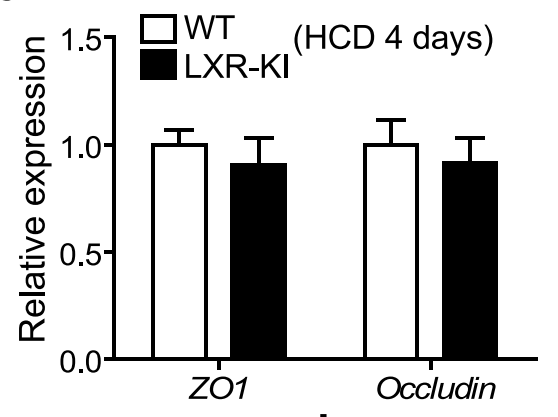

H Serum cholesterol (HCD 4 days)

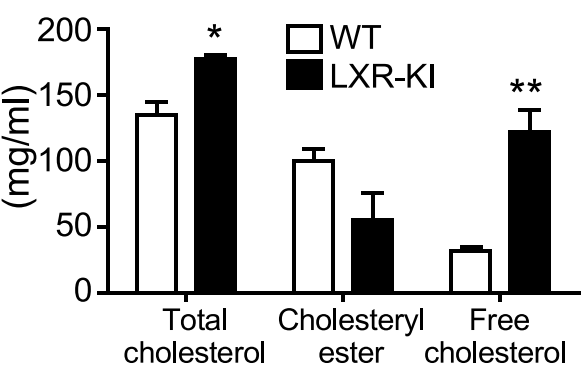

Fig. 5. Activation of LXR $\alpha$ increases intestinal cholesterol absorption even in the presence of the Npc1l1 inhibitor ezetimibe and precedes gut toxicity. (A) Basal cholesterol absorption in WT and LXR-KI male mice fed chow diet. (B) Expression of Niemann-Pick C1-like 1 (Npc1l1) in duodenum/proximal jejunum. (C) Serum cholesterol levels expressed as total, cholesteryl ester, and free cholesterol in male mice fed chow diet $(n=4)$. (D) Cholesterol absorption male mice fed HCD for 4 or 12 days in the absence or presence of daily ezetimibe $(10 \mathrm{mg} / \mathrm{kg})$ treatment. (E) Serum total cholesterol levels in male mice fed an HCD in the absence or presence of ezetimibe treatment. (F) H\&E staining of duodenal sections in male mice fed an HCD for 4 days. (G) The intestinal mRNA expression of tight junction protein genes in mice fed HCD for 4 days. $(\mathrm{H})$ Serum cholesterol levels expressed as total, cholesteryl ester, and free cholesterol in male mice fed HCD for 4 days $(n=4-6)$. (I and J) Fecal concentrations of cholesterol (I) and triglycerides (J) in chow-fed mice. ${ }^{*} P<0.05 ; * * P<0.01 ;{ }^{* * *} P<0.001$, n.s., statistically not significant, LXR-KI vs. WT, or the comparisons are labeled.

was lower in the LXR-KI mice (Fig. 5A), likely owing to decreased basal expression of Niemann-Pick C1-Like 1 (Npc1l1) (Fig. 5B). Npc1l1 is one of the best characterized intestinal cholesterol absorption transporters, whose expression is known to be suppressed by LXR (Duval et al., 2006). NPC1L1 is also the therapeutic target of the clinical drug 
ezetimibe, a pharmacological inhibitor of NPC1L1 that inhibits dietary cholesterol absorption (Van Heek et al., 1997); however, this modest reduction in basal cholesterol absorption had little effect on the circulating levels of cholesterol in chowfed mice (Fig. 5C), likely because of a low content of cholesterol in normal chow; however, when the mice were challenged with HCD, cholesterol absorption was markedly enhanced in the LXR-KI mice as early as after 4 days of HCD feeding and even in the presence of ezetimibe treatment $(10 \mathrm{mg} / \mathrm{kg}$ per day) (Fig. 5D). There was a modest inhibitory effect of ezetimibe on the cholesterol absorption in the LXR-KI mice, which was most likely due to residual expression and activity of intestinal Npc1l1 in these mice; however, the inhibitory effect of ezetimibe was estimated to be minor in the scale of increased cholesterol absorption in the LXR-KI mice. Under the HCD feeding, the serum cholesterol level in the WT mice was lowered by ezetimibe, as expected, but the same drug had little effect on the serum level of total cholesterol in the LXR-KI mice (Fig. 5E), suggesting that the HCD-fed LXR-KI mice had an increased cholesterol absorption even in the presence of a Npc1l1 inhibitor. The increased intestinal cholesterol absorption in the HCD-fed LXR-KI mice preceded the gut toxicity because at 4 days of HCD feeding, when cholesterol absorption had already increased in a statistically significant manner
(Fig. 5D), little appreciable histologic damage was seen in the intestine of the LXR-KI mice (Fig. $5 \mathrm{~F}$ ) or changes in mRNA expression of $\mathrm{ZO} 1$ and Occludin (Fig. 5G). The increased serum levels of total cholesterol and free cholesterol also preceded the gut toxicity (Fig. $5 \mathrm{H}$ ). When the fecal concentrations of lipids were measured in chow-fed mice, we found the fecal concentrations of cholesterol did not differ between WT and LXR-KI mice (Fig. 5I), but the fecal triglyceride level was high in LXR-KI mice (Fig. 5J).

Activation of LXR Stimulates Cholesterol Uptake in Colon Cancer Cells. Stimulation of cholesterol uptake by LXR activation was also observed in colon cancer cells. The human colon cancer HCT116 cells express functional LXRs because treatment of these cells with GW3965 induced the expression of LXR target genes, such as stearoyl-CoA desaturase-1 and fatty acid synthase (Fig. 6A). To measure the cholesterol uptake, HCT116 cells were treated with vehicle or $1 \mu \mathrm{M}$ of GW3965 for 24 hours before subjecting the cells to cholesterol uptake assay using the cholesterol fluorescent analog NBD-cholesterol. As shown in Fig. 6B, treatment with GW3965 increased the uptake of NBDcholesterol compared with the vehicle control. The effect of GW3965 on the uptake of NBD-cholesterol was abolished when both LXR $\alpha$ and $\beta$ were knocked down by siRNA
A

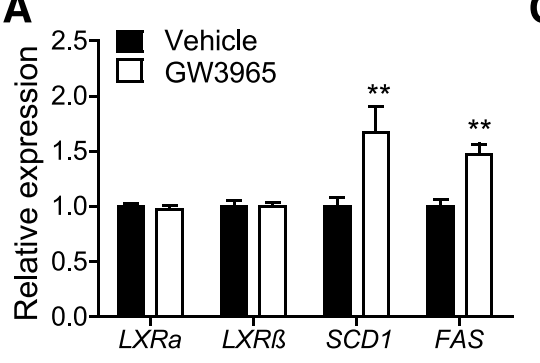

B

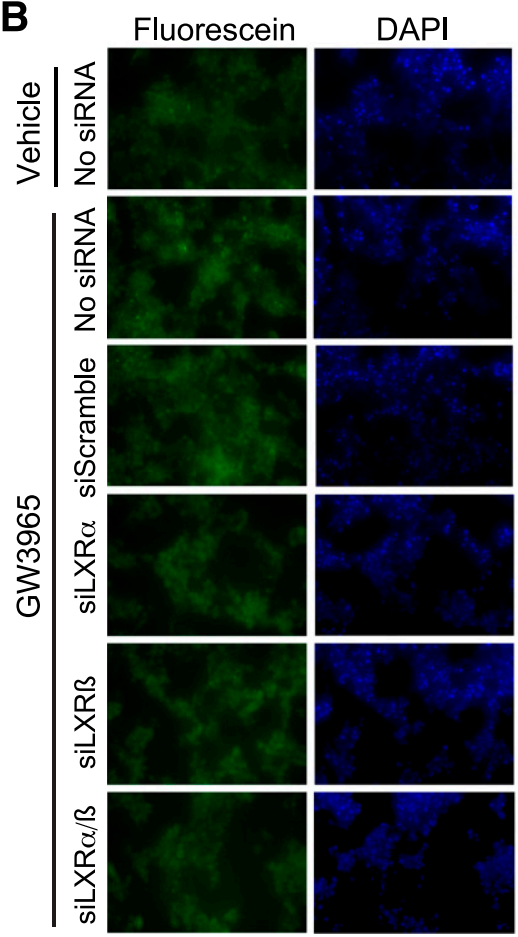

C

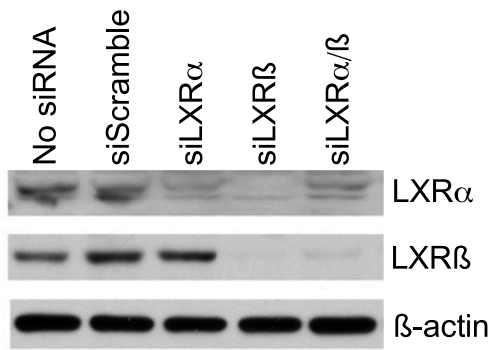

D
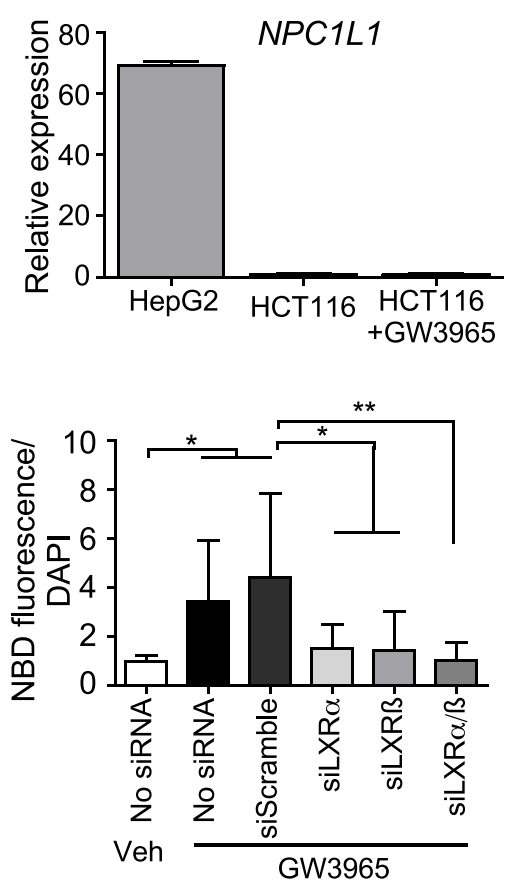

Fig. 6. Activation of LXR stimulates cholesterol uptake in colon cancer cells. (A) mRNA expression of LXRs and their target genes in HCT116 cells treated with vehicle or $1 \mu \mathrm{M}$ GW3965 was measured by real-time PCR. (B) The uptake of fluorescent NBD-cholesterol in HCT116 cells treated with vehicle or GW3965 $(1 \mu \mathrm{M})$. The GW3965-treated cells include those transfected with scramble siRNA, siLXR $\alpha$ and/or siLXR $\beta$. Shown on the right are the quantifications of the NBD fluorescence intensity normalized by DAPI staining and quantified by Image J. (C) The efficiency of LXR siRNA knock-down was verified by Western blotting. (D) The mRNA expression of NPC1LI in HepG2 and HCT116 cells was measured by real-time PCR. $* P<0.05 ; * * P<0.01$, GW3965 vs. vehicle (A) or the comparisons are labeled. 
(Fig. 6B). The efficiency of LXR knockdown was confirmed by Western blotting (Fig. 6C). The expression of NPC1L1 was barely detectable in HCT116 cells (Fig. 6D), suggesting that the GW3965 effect on the uptake of NBD-cholesterol in these cells was independent of NPC1L1.

\section{Discussion}

One of the most surprising findings in this study is that activation of LXR $\alpha$ promotes intestinal cholesterol uptake. To our knowledge, NPC1L1 is the only known intestinal cholesterol absorption transporter that can be pharmacologically inhibited by ezetimibe (Van Heek et al., 1997) or transcriptionally suppressed by $\operatorname{LXR} \alpha$ (Duval et al., 2006). The latter mechanism is yet to be clinically explored because of the prolipogenic side effect of LXR $\alpha$ activation in the liver (Schultz et al., 2000). Nevertheless, harnessing the LXR-mediated RCT pathway to prevent or treat arthrosis remains to be an attractive idea. The challenge, as suggested by previous studies by us and others, is that the LXR $\alpha$ biology can be highly conditional.

In the current study, while examining the effect of chronic activation of LXR under the HCD condition, we discovered an unexpected function of $\mathrm{LXR} \alpha$ in the gut. Under HCD, genetic or pharmacologic activation of $\operatorname{LXR} \alpha$ induced gut toxicity and systemic toxicity, causing a dramatic weight loss. This phenotype was strictly diet-dependent because neither the chow-fed LXR-KI mice nor the GW3965-treated WT mice under chow diet exhibited appreciable toxicity. An intriguing observation was that the HCD-triggered pathology was small intestine-specific because there was little pathology in the livers of the HCD-fed LXR-KI mice. The apoptotic or necrotic changes in the enterocytes may be attributed to a higher free cholesterol content found in the intestinal cells. Unlike the cholesteryl esters, the free cholesterol is soluble and therefore potentially toxic to the phospholipid membranes of the endoplasmic reticulum (Tabas, 2002). Our results strongly suggested that the gut toxicity was a result of increased intestinal cholesterol uptake and increased concentrations of free cholesterol in the enterocytes because increased intestinal cholesterol absorption preceded the gut toxicity. The mRNA expression of the tight-junction protein genes Occludin and $\mathrm{ZO} 1$ did not change after 4 days of HCD feeding, at which the cholesterol absorption has already increased in the LXR-KI mice, suggesting that LXR does not directly regulate the expression of Occludin and ZO1; however, we cannot exclude the possibility that disruption of the integrity of the intestinal tights junction may have contributed to the increased cholesterol absorption in the LXR-KI mice, especially once the gut toxicity and disruption of intestinal villus structure have occurred. Cholesterol accumulation in the intestine of LXR-KI mice was unlikely attributable to a decreased transintestinal excretion of cholesterol because the expression of intestinal cholesterol efflux transporters Abcg5/8 was increased in the LXR-KI mice regardless of the diet treatment. The HCD contains $0.5 \%$ cholic acid, which is necessary for the absorption of cholesterol from a HCD (Chiang et al., 1998; Woollett et al., 2004). It is unlikely the gut toxicity in HCD-fed LXR-KI mice was due to the cholic acid because HCD failed to cause appreciable toxicity in the WT mice. Moreover, a control diet contains the same level of cholic acid but without excess cholesterol did not cause gut toxicity in either the WT or LXR-KI mice (data not shown).

Another surprising finding is the rise of intestinal and circulating levels of cholesterol in HCD-fed LXR-KI mice despite the reduced basal expression of Npc1l1 in these mice. The reduced expression of Npc1l1 was expected because this transporter is known to be transcriptionally suppressed by LXR $\alpha$ (Duval et al., 2006). In addition, high cholesterol feeding by itself can also suppress the expression of intestinal Npc1l1 in mice (Kawase et al., 2016). Although there are reports suggesting that LXR activation inhibited intestinal cholesterol absorption by suppressing Npc111 (Altmann et al., 2004; Duval et al., 2006), it has been controversial whether the LXR responsive suppression of Npc1l1 is sufficient to cause a suppression of cholesterol uptake. For example, a report showed that GW3965 can reduce the intestinal expression of Npc111, but it was not sufficient to affect the intestinal cholesterol absorption under aN HCD (Kannisto et al., 2014). Another study reported that treatment with GW3965 can increase the intestinal cholesterol absorption in mice despite a reduced intestinal expression of Npc1l1 (Hu et al., 2012). The Npc1l1-indepdent absorption of cholesterol in the LXR-KI mice was further supported by our observation that the cholesterol absorption was increased in the LXR-KI mice, even in the presence of ezetimibe, a pharmacological inhibitor of Npc1l1 (Van Heek et al., 1997).

It is interesting to note that the effect of transgenic activation of $\operatorname{LXR} \alpha$ on cholesterol absorption was dietdependent: decreased absorption under chow diet and increased absorption when the mice were challenged with HCD. The decreased absorption under chow diet was consistent with a report in which the authors showed that intestinal-specific transgenic activation of LXR $\alpha$ led to decreased intestinal cholesterol absorption, improved lipoprotein profile, and increased RCT in vivo (Lo Sasso et al., 2010). It remains to be seen whether the intestinal-specific LXR transgenic mice reported by Lo Sasso and colleagues will exhibit gut toxicity or cholesterol accumulation when challenged with HCD. Nevertheless, our results presented yet another example that the LXR $\alpha$ biology can be highly context-dependent. The conditional promotion of cholesterol absorption by LXR activation may not be limited to the intestine and enterocytes because increased uptake of highdensity lipoprotein-derived cholesteryl esters has been reported in human macrophages upon LXR activation (Bultel et al., 2008).

In summary, we report an unexpected function of LXR $\alpha$ in intestinal cholesterol absorption and gut toxicity. The increased cholesterol absorption in the LXR $\alpha$-activated intestine appeared to be independent of Npc1l1 and likely is mediated by a novel, yet-to-be-defined mechanism.

\section{Acknowledgments}

We thank Dr. Li Gao for helpful comments and discussions.

\section{Authorship Contributions}

Participated in research design: Garbacz, Uppal, Yan, Huang, Xie. Conducted experiments: Garbacz, Uppal, Yan, Xu, Ren, Stolz. Performed data analysis: Garbacz, Xie.

Wrote or contributed to the writing of the manuscript: Garbacz, Huang, Xie. 


\section{References}

Altmann SW, Davis HR, Jr, Zhu LJ, Yao X, Hoos LM, Tetzloff G, Iyer SP, Maguire M, Golovko A, Zeng M, et al. (2004) Niemann-Pick C1 Like 1 protein is critical for intestinal cholesterol absorption. Science 303:1201-1204.

Beltroy EP, Richardson JA, Horton JD, Turley SD, and Dietschy JM (2005) Cholesterol accumulation and liver cell death in mice with Niemann-Pick type C disease. Hepatology 42:886-893.

Bonamassa B and Moschetta A (2013) Atherosclerosis: lessons from LXR and the intestine. Trends Endocrinol Metab 24:120-128.

Breevoort SR, Angdisen J, and Schulman IG (2014) Macrophage-independent regulation of reverse cholesterol transport by liver X receptors. Arterioscler Thromb Vasc Biol 34:1650-1660.

Bultel S, Helin L, Clavey V, Chinetti-Gbaguidi G, Rigamonti E, Colin M, Fruchart JC, Staels B, and Lestavel S (2008) Liver X receptor activation induces the uptake of cholesteryl esters from high density lipoproteins in primary human macrophages. Arterioscler Thromb Vasc Biol 28:2288-2295.

Calon A, Gross I, Lhermitte B, Martin E, Beck F, Duclos B, Kedinger M, Duluc I, Domon-Dell C, and Freund JN (2007) Different effects of the Cdx1 and Cdx2 homeobox genes in a murine model of intestinal inflammation. Gut 56:1688-1695.

Calpe-Berdiel L, Rotllan N, Fiévet C, Roig R, Blanco-Vaca F, and Escolà-Gil JC (2008) Liver X receptor-mediated activation of reverse cholesterol transport from macrophages to feces in vivo requires ABCG5/G8. J Lipid Res 49:1904-1911.

Chawla A, Repa JJ, Evans RM, and Mangelsdorf DJ (2001) Nuclear receptors and lipid physiology: opening the X-files. Science 294:1866-1870.

Chiang MT, Chen YC, and Huang AL (1998) Plasma lipoprotein cholesterol levels in rats fed a diet enriched in cholesterol and cholic acid. Int $J$ Vitam Nutr Res 68 328-334.

Davis HR, Jr, Zhu LJ, Hoos LM, Tetzloff G, Maguire M, Liu J, Yao X, Iyer SP, Lam MH, Lund EG, et al. (2004) Niemann-Pick C1 Like 1 (NPC1L1) is the intestinal phytosterol and cholesterol transporter and a key modulator of whole-body cholesterol homeostasis. J Biol Chem 279:33586-33592.

Duval C, Touche V, Tailleux A, Fruchart JC, Fievet C, Clavey V, Staels B, and Lestavel S (2006) Niemann-Pick C1 like 1 gene expression is down-regulated by LXR activators in the intestine. Biochem Biophys Res Commun 340:1259-1263.

Folch J, Lees M, and Sloane Stanley GH (1957) A simple method for the isolation and purification of total lipides from animal tissues. J Biol Chem 226:497-509.

Gong H, He J, Lee JH, Mallick E, Gao X, Li S, Homanics GE, and Xie W (2009) Activation of the liver $\mathrm{X}$ receptor prevents lipopolysaccharide-induced lung injury. $J$ Biol Chem 284:30113-30121.

Hu X, Steffensen KR, Jiang ZY, Parini P, Gustafsson JA, Gåfvels M, and Eggertsen G (2012) LXR $\beta$ activation increases intestinal cholesterol absorption, leading to an atherogenic lipoprotein profile. J Intern Med 272:452-464.

Jakobsson T, Treuter E, Gustafsson JA, and Steffensen KR (2012) Liver X receptor biology and pharmacology: new pathways, challenges and opportunities. Trends Pharmacol Sci 33:394-404.

Joseph SB, McKilligin E, Pei L, Watson MA, Collins AR, Laffitte BA, Chen M, Noh G, Goodman J, Hagger GN, et al. (2002) Synthetic LXR ligand inhibits the development of atherosclerosis in mice. Proc Natl Acad Sci USA 99:7604-7609.

Kannisto K, Gåfvels M, Jiang ZY, Slätis K, Hu X, Jorns C, Steffensen KR, and Eggertsen G (2014) LXR driven induction of HDL-cholesterol is independent of intestinal cholesterol absorption and ABCA1 protein expression. Lipids 49:71-83.

Kawase A, Araki Y, Ueda Y, Nakazaki S, and Iwaki M (2016) Impact of a highcholesterol diet on expression levels of Niemann-Pick C1-like 1 and intestinal transporters in rats and mice. Eur $J$ Drug Metab Pharmacokinet 41:457-463.

Komati R, Spadoni D, Zheng S, Sridhar J, Riley KE, and Wang G (2017) Ligands of therapeutic utility for the liver X receptors. Molecules 22 (1):88.

Kruth HS (1984) Localization of unesterified cholesterol in human atherosclerotic lesions. Demonstration of filipin-positive, oil-red-O-negative particles. Am J Pathol 114:201-208.

Lo Sasso G, Murzilli S, Salvatore L, D’Errico I, Petruzzelli M, Conca P, Jiang ZY, Calabresi L, Parini P, and Moschetta A (2010) Intestinal specific LXR activation stimulates reverse cholesterol transport and protects from atherosclerosis. Cell Metab 12:187-193.
Maxfield FR and Tabas I (2005) Role of cholesterol and lipid organization in disease. Nature 438:612-621.

Mitrura BM and Rawnsley MH (1981) Clinical Biochemical and Haematological Reference Values in Normal Experimental Animals and Normal Humans, Mosby, New York.

Mutemberezi V, Guillemot-Legris O, and Muccioli GG (2016) Oxysterols: from cholesterol metabolites to key mediators. Prog Lipid Res 64:152-169.

Nakao A, Kimizuka K, Stolz DB, Neto JS, Kaizu T, Choi AM, Uchiyama T, Zuckerbraun BS, Nalesnik MA, Otterbein LE, et al. (2003) Carbon monoxide inhalation protects rat intestinal grafts from ischemia/reperfusion injury. Am J Pathol 163: 1587-1598.

Ou Z, Jiang M, Hu B, Huang Y, Xu M, Ren S, Li S, Liu S, Xie W, and Huang M (2014) Transcriptional regulation of human hydroxysteroid sulfotransferase SULT2A1 by LXR $\alpha$. Drug Metab Dispos 42:1684-1689.

Peet DJ, Turley SD, Ma W, Janowski BA, Lobaccaro JM, Hammer RE, and Mangelsdorf DJ (1998) Cholesterol and bile acid metabolism are im paired in mice lacking the nuclear oxysterol receptor LXR alpha. Cell 93: 693-704.

Repa JJ, Berge KE, Pomajzl C, Richardson JA, Hobbs H, and Mangelsdorf DJ (2002) Regulation of ATP-binding cassette sterol transporters ABCG5 and ABCG8 by the liver X receptors alpha and beta. J Biol Chem 277:18793-18800.

Schultz JR, Tu H, Luk A, Repa JJ, Medina JC, Li L, Schwendner S, Wang S, Thoolen M, Mangelsdorf DJ, et al. (2000) Role of LXRs in control of lipogenesis. Genes Dev 14:2831-2838.

Shakir KM, Sundaram SG, and Margolis S (1978) Lipid synthesis in isolated intestinal cells. J Lipid Res 19:433-442.

Tabas I (2002) Consequences of cellular cholesterol accumulation: basic concepts and physiological implications. J Clin Invest 110:905-911.

Ulluwishewa D, Anderson RC, McNabb WC, Moughan PJ, Wells JM, and Roy NC (2011) Regulation of tight junction permeability by intestinal bacteria and dietary components. J Nutr 141:769-776.

Uppal H, Saini SP, Moschetta A, Mu Y, Zhou J, Gong H, Zhai Y, Ren S, Michalopoulos GK, Mangelsdorf DJ, et al. (2007) Activation of LXRs prevents bile acid toxicity and cholestasis in female mice. Hepatology 45:422-432.

van der Veen JN, van Dijk TH, Vrins CL, van Meer H, Havinga R, Bijsterveld K Tietge UJ, Groen AK, and Kuipers F (2009) Activation of the liver X receptor stimulates trans-intestinal excretion of plasma cholesterol. $J$ Biol Chem 284: 19211-19219.

Van Heek M, France CF, Compton DS, McLeod RL, Yumibe NP, Alton KB, Sybertz EJ, and Davis HR, Jr (1997) In vivo metabolism-based discovery of a potent cholesterol absorption inhibitor, SCH58235, in the rat and rhesus monkey through the identification of the active metabolites of SCH48461. J Pharmacol Exp Ther 283: 157-163.

Werder M, Han CH, Wehrli E, Bimmler D, Schulthess G, and Hauser H (2001) Role of scavenger receptors SR-BI and CD36 in selective sterol uptake in the small intestine. Biochemistry 40:11643-11650.

Willy PJ, Umesono K, Ong ES, Evans RM, Heyman RA, and Mangelsdorf DJ (1995) LXR, a nuclear receptor that defines a distinct retinoid response pathway. Genes Dev 9:1033-1045.

Woollett LA, Buckley DD, Yao L, Jones PJ, Granholm NA, Tolley EA, Tso P, and Heubi JE (2004) Cholic acid supplementation enhances cholesterol absorption in humans. Gastroenterology 126:724-731.

Zelcer N, Hong C, Boyadjian R, and Tontonoz P (2009) LXR regulates cholesterol uptake through Idol-dependent ubiquitination of the LDL receptor. Science 325 : 100-104.

Zhao C and Dahlman-Wright K (2010) Liver X receptor in cholesterol metabolism. $J$ Endocrinol 204:233-240.

Address correspondence to: Wen Xie, Center for Pharmacogenetics and Department of Pharmaceutical Sciences, 306 Salk Pavilion, University of Pittsburgh, Pittsburgh, PA 15261. E-mail: wex6@pitt.edu 